\title{
Growth and Bioactive Compound Synthesis in Cultivated Lettuce Subject to Light-quality Changes
}

Ki-Ho Son, Jin-Hui Lee, Youngjae Oh, Daeil Kim, and Myung-Min Oh ${ }^{\mathbf{1}}$ Division of Animal, Horticultural and Food Sciences, Chungbuk National University, Cheongju 28644, South Korea; and Brain Korea 21 Center for Bio-Resource Development, Chungbuk National University, Cheongju 28644, South Korea

\section{Byung-Chun In}

Department of Bioindustry and Bioresources Engineering, Sejong University, Seoul 05006, South Korea

Additional index words. chalcone synthase, Lactuca sativa, phenylalanine ammonia-lyase, plant factory

\begin{abstract}
This study aimed to determine the effect of changes in light quality on the improvement of growth and bioactive compound synthesis in red-leaf lettuce (Lactuca sativa L. 'Sunmang') grown in a plant factory with electrical lighting. Lettuce seedlings were subjected to 12 light treatments combining five lighting sources: red $(R ; 655 \mathbf{~ n m})$, blue $(B ; 456 \mathrm{~nm})$, and different ratios of red and blue light combined with three lightemitting diodes [LEDs (R9B1, R8B2, and R6B4)]. Treatments were divided into control (continuous irradiation of each light source for 4 weeks), monochromatic (changing from $R$ to $B$ at 1,2 , or 3 weeks after the onset of the experiments), and combined (changing from R9B1 to R8B2 or R6B4 at 2 or 3 weeks after the onset of the experiments). Growth and photosynthetic rates of lettuce increased with increasing ratios of red light, whereas chlorophyll and antioxidant phenolic content decreased with increasing ratios of red light. Individual phenolic compounds, including chlorogenic, caffeic, chicoric, and ferulic acids, and kaempferol, showed a similar trend to that of total phenolics. Moreover, transcript levels of phenylalanine ammonia-lyase $(P A L)$ and chalcone synthase $(C H S)$ genes were rapidly upregulated by changing light quality from red to blue. Although the concentration of bioactive compounds in lettuce leaves enhanced with blue light, their contents per lettuce plant were more directly affected by red light, suggesting that biomass as well as bioactive compounds' accumulation should be considered to enhance phytochemical production. In addition, results suggested that growth and antioxidant phenolic compound synthesis were more sensitive to monochromatic light than to combined light variations. In conclusion, the adjustment of light quality at a specific growth stage should be considered as a strategic tool for improving crop yield, nutritional quality, or both in a plant factory with electrical lighting.
\end{abstract}

Closed-type plant production systems incur additional costs when using electrical light sources instead of sunlight, which requires an increase in crop yield and quality to obtain economic feasibility for crop cultivation (Piovene et al., 2015). Therefore, a systematic approach is necessary for developing technology that controls light conditions as these influence crop yield and quality factors. Light-emitting diodes, commonly used as electrical light sources for closed-type plant production systems, can generate optimal light conditions for improving crop yield because of their reduced energy consumption and control of light quality, intensity, and period (Massa et al., 2008). The production of phytochemicals, which are considered major indicators of crop quality, can be enhanced

Received for publication 17 Nov. 2016. Accepted for publication $30 \mathrm{Jan} .2017$.

${ }^{1}$ Corresponding author. E-mail: moh@cbnu.ac.kr. through LED irradiation (Bian et al., 2014; Lefsrud et al., 2008; Son et al., 2012). However, most closed-type plant production systems have only been performing a passive light control, such as fixed light quality and intensity, rather than changing light quality using LEDs.

Recently, Bian et al. (2014) reported that the accumulation of phytochemicals in vegetable crops, such as lettuce, cucumber, tomato, radish, and spinach, depended on light quality and delineated the effects of phytochemicals under different light quality and intensity. Light quality, in particular, had a major impact on phytochemicals: carotenoids ( $\beta$-carotene, lutein), phenolics (anthocyanins, flavonoids), and vitamin $\mathrm{C}$ respond differently to LED quality in terms of ultraviolet-A (315-380 nm), blue (425-490 $\mathrm{nm})$, green $(490-550 \mathrm{~nm})$, red $(625-700 \mathrm{~nm})$, and far red (700-740 nm) radiation in green vegetables, tomato, cucumber, and sweet pepper (Olle and Viršilè, 2013). Ultraviolet, which has high energy and short wavelength, is known to promote biosynthesis of phytochemicals by inducing plants' defense mechanisms as this radiation usually stresses the plant (Gartia et al., 2003). Among the visible spectrums, red and blue light are important for photosynthesis and have often been used in plant research and commercial production. According to our previous studies, red and blue LEDs effectively enhanced lettuce ( $L$. sativa L.) plant growth and the synthesis of secondary metabolites, respectively, although the response to light quality depended on lettuce variety (cultivar) (Son et al., 2012; Son and Oh, 2013, 2015).

In general, plants allocate or distribute their resources between growth and development, which was described in the so-called carbon-nutrient balance model (Bryant et al., 1983) and growth-differentiation balance framework (Herms and Mattson, 1992). This resource partitioning might affect the synthesis of secondary metabolites differently during all growth stages. Therefore, determining a reasonable harvest time and controlling the growth environment are necessary to obtain mature plants with maximal concentrations of secondary metabolites. In this aspect, the narrow-bandwidth light provided by LEDs may affect the marketable value of crops, and the irradiation protocol might be a strategy in crops' production technology. Carvalho and Folta (2014) suggested that light change during sprouting of kale seeds affected growth and development and could be a method to produce value-added crops. Similarly, light shift using blue and red LEDs in the production of lettuce and basil was effective in promoting plant growth or phenolic content (Jishi et al., 2016; Taulavuori et al., 2016). Based on these premises, the present study aims to determine the effect of changing light quality on growth and secondary metabolites of lettuce at a particular stage, providing basic information for improving the content of secondary metabolites. Changes in growth rate and secondary metabolite contents of lettuces were monitored to test if the content of bioactive compounds per lettuce plant would be higher in plants irradiated with a light quality that improved growth rate (mainly red light) followed by a light quality that improved secondary metabolites production (mainly blue light) than it would be in plants continuously irradiated by red or blue light.

\section{Materials and Methods}

\section{Plant material and growing conditions}

Red-leaf lettuce seeds ('Sunmang'; Nongwoo Bio, Suwon, Korea) were sown in a plug tray (32 $\mathrm{mL} /$ cell) with horticultural growing medium (Myung-Moon; Dongbu Hannong, Seoul, Korea). The plug tray was placed on a shelf and the following growth conditions were maintained for $18 \mathrm{~d}$ : air temperature, $20{ }^{\circ} \mathrm{C}$; relative humidity $(\mathrm{RH}), 60 \% ; \mathrm{CO}_{2}$ concentration, $1000 \mu \mathrm{mol} \cdot \mathrm{mol}^{-1}$; photosynthetic photon flux density $(P P F D)$ generated by fluorescent lamps, $119 \pm 5 \mu \mathrm{mol} \cdot \mathrm{m}^{-2} \cdot \mathrm{s}^{-1}$; 
and light period, $12 \mathrm{~h}$. Twenty-five seedlings were allocated to each light-quality treatment (one seedling per pot; pots $7 \mathrm{~cm} \mathrm{~L} \times 7 \mathrm{~cm} \mathrm{~W} \times$ $7.4 \mathrm{~cm} \mathrm{H}$ ) and transferred to other shelves in the same growing room. Lettuce plants were subirrigated with distilled water every $2-3 \mathrm{~d}$ and with a nutrient solution (17.3 N: 4.0 P: 8.0 $\mathrm{K}, \mathrm{pH} 5.5$, electrical conductivity $1.16 \mathrm{dS} \cdot \mathrm{m}^{-1}$ ) once a week for 4 weeks after transplanting. All lettuce plants were grown in a plant factory $(4 \mathrm{~m} \mathrm{~L} \times 2 \mathrm{~m} \mathrm{~W} \times 3 \mathrm{~m} \mathrm{H})$ under controlled environmental conditions (air temperature, $20{ }^{\circ} \mathrm{C}$; $\mathrm{RH}, 60 \% ; \mathrm{CO}_{2}$ concentration, 1000 $\mu \mathrm{mol} \cdot \mathrm{mol}^{-1}$; and light period, $12 \mathrm{~h}$ ) for 4 weeks after transplanting. Identical LED irradiation conditions $\left(P P F D, 151 \pm 4 \mu \mathrm{mol} \cdot \mathrm{m}^{-2} \cdot \mathrm{s}^{-1}\right)$ and light period $(12 \mathrm{~h})$ were applied to each treatment. Lettuce plants were systematically rearranged every day to avoid a disproportionate distribution of irradiation.

\section{Light-quality treatment}

Five plate-type $(48 \mathrm{~cm} \mathrm{~L} \times 48 \mathrm{~cm} \mathrm{~W})$ monochromatic (blue and red) and combined (red/blue ratios based on the number of the LED chips; R9B1, R8B2, and R6B4) LEDs were used as lighting sources in this study. The wavelengths of red (R, Bright LED Electronics, Seoul, Korea) and blue (B, ITSwell, Incheon, Korea) LEDs were 655 and 456 $\mathrm{nm}$, respectively. The spectral characteristics of each lighting source were measured and adjusted at nine points (one in the center and eight on the edges of the tray) using an LI-1800 spectroradiometer and an LI-190 quantum sensor (both LI-COR Inc., Lincoln, NE) as described in Son and Oh (2013).

To determine the effect of different light combinations on growth and secondary metabolism of lettuce, 12 treatments were applied using monochromatic and combined LEDs. This experimental design was based on the results of previous studies using monochromatic LEDs (Son et al., 2012) and several combinations of red and blue LEDs (Son and Oh, 2013) (Table 1). In monochromatic treatments, red LEDs were selected to promote growth in the early growth stage, and blue LEDs were selected to increase secondary metabolites production in the next red irradiation. Among the combined LEDs, R9B1 was selected to promote growth, whereas R8B2 and R6B4 were selected to increase secondary metabolites content and concentration, respectively, based on the results of our previous study (Son and Oh, 2013). Within monochromatic treatments, irradiation changed from red to blue at 1 (M1), 2 (M2), or 3 (M3) weeks after the onset of the experiment. The combined-type LED irradiation changed from $\mathrm{R} 9 \mathrm{~B} 1$ to R8B2 or R6B4 at 2 (C1 and $\mathrm{C} 3$ ) or 3 (C2 and $\mathrm{C} 4)$ weeks after the onset of the treatment. All treatments were applied to lettuce plants within the same area of the plant factory and at the same time.

\section{Growth characteristics}

Biomass. Growth characteristics, such as the fresh and dry weight (DW) of shoots and roots, leaf number, and leaf area, were measured at 1-week intervals after the onset of the treatments. The fresh weight of shoots and roots was determined using a Si-234 electronic scale (Denver Instruments, Bohemia, NY). To obtain shoot and root DW, these were placed for $3 \mathrm{~d}$ at $70^{\circ} \mathrm{C}$ in a VS- 120203 oven (Vision Scientific, Daejeon, Korea) and then weighed. Leaf area was measured using a leaf area meter (LI-3000A; Li-COR Inc., Lincoln, NE).

Chlorophyll content and photosynthetic rate. Chlorophyll content was measured every week as the SPAD value determined by a portable chlorophyll meter (SPAD-502; KONICA MINOLTA, Osaka, Japan). The net photosynthesis of the lettuces grown under 12 treatments was measured using the LI-6400 portable photosynthesis system (Li-COR Inc., Lincoln, NE) for 3-4 weeks after the onset of the treatments. To precisely determine the effect of light quality, a 6400-08 clear chamber (Li-COR Inc., Lincoln, NE) was used as this chamber can transmit LED light into the leaf samples placed within it. The conditions of flow rate, $\mathrm{CO}_{2}$ levels, $P P F D$, and leaf temperature within each leaf sample cuvette were maintained at $350 \mu \mathrm{mol} \cdot \mathrm{s}^{-1}, 1000$ $\mu \mathrm{mol} \cdot \mathrm{mol}^{-1}, 150 \pm 5 \mu \mathrm{mol} \cdot \mathrm{m}^{-2} \cdot \mathrm{s}^{-1}$, and $20{ }^{\circ} \mathrm{C}$, respectively. SPAD values and photosynthetic rates were determined using the third leaf from the top of each plant.

Projected leaf area (PLA). To determine the leaf area index of the lettuce plants grown under each treatment, PLAs were obtained in the image analysis software LIA32 (K. Yamamoto, Nagoya University, Nagoya,

Table 1. The 12 light treatments applied, based on monochromatic (M) red (R) and blue (B) LEDs, or on combined (C) LEDs, and the fraction of blue and red light within each treatment.

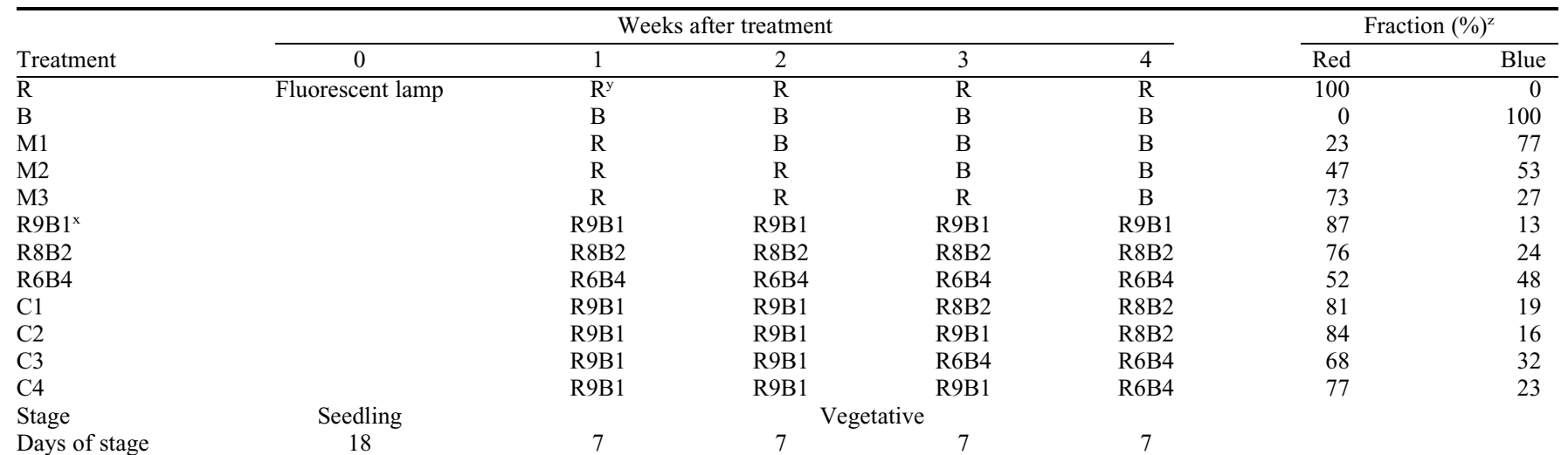

LED $=$ light-emitting diode.

${ }^{\mathrm{z}}$ Fractions of integrated blue and red wavelengths in terms of photosynthetic photon flux density at each treatment during all stages.

${ }^{\mathrm{y}}$ Irradiated LED qualities at each stage $(\mathrm{R}=$ red or $\mathrm{B}=$ blue $)$.

${ }^{\mathrm{x}}$ Ratios of red and blue LEDs based on the number of LED chips.

Table 2. Primers and cycle conditions used in the quantitative real-time PCR and their target gene.

\begin{tabular}{|c|c|c|c|c|c|}
\hline \multirow[b]{2}{*}{ Gene $^{z}$} & \multirow[b]{2}{*}{ Accession no. } & \multirow[b]{2}{*}{ Primer sequence } & \multirow[b]{2}{*}{ Length (mer) } & \multicolumn{2}{|c|}{ Cycle conditions } \\
\hline & & & & $\mathrm{Tm}^{\mathrm{y}}\left({ }^{\circ} \mathrm{C}\right)$ & No. of cycles \\
\hline \multirow[t]{2}{*}{$\overline{L s P A L}$} & AF299330.1 & F: CAAGGGAAGCCGGAGTTTAC & $20 / 20$ & 52 & 40 \\
\hline & & R: CTGGAAACGTCGATCAATGG & & & \\
\hline \multirow[t]{2}{*}{$\mathrm{LsCHS}$} & AB525909.1 & F: CTCACTAAGCTCCTCGGCCT & $20 / 20$ & 55 & 40 \\
\hline & & R: TTGTCCAACGAGGGAATCAA & & & \\
\hline \multirow[t]{2}{*}{ Lsactin } & AY260165.1 & F: AGCAACTGGGATGACATGGA & $20 / 20$ & & \\
\hline & & R: GGGTTGAGAGGTGCCTCAGT & & & \\
\hline
\end{tabular}

${ }^{\mathrm{z}}$ LsPAL (Lactuca sativa phenylalanine ammonia-lyase mRNA), LsCHS (Lactuca sativa chalcone synthase), and Lsactin (Lactuca sativa actin mRNA).

${ }^{\mathrm{y}}$ Annealing temperature. 


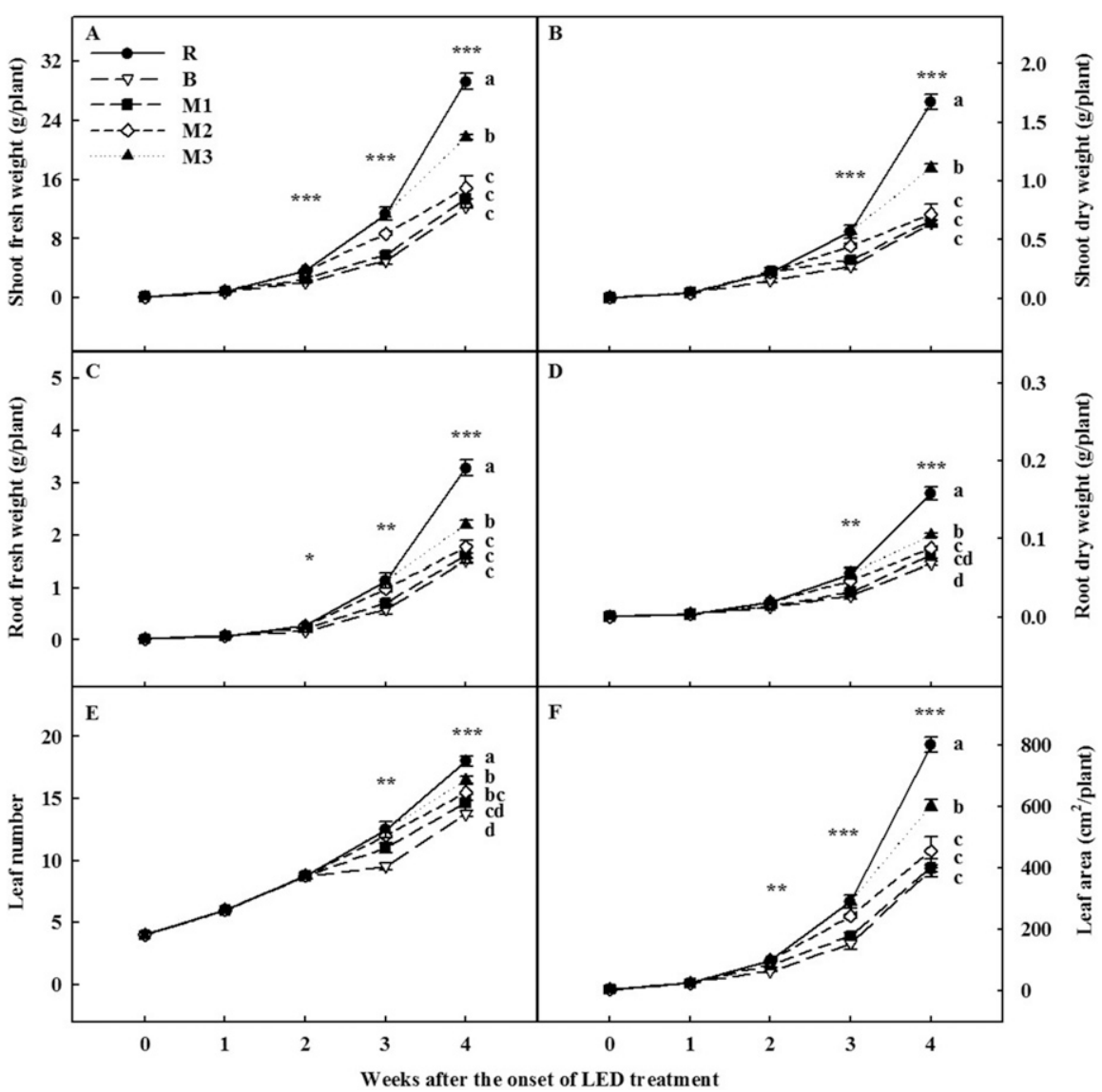

Fig. 1. Growth of shoots $(\mathbf{A}$ and $\mathbf{B})$, roots $(\mathbf{C}$ and $\mathbf{D})$, and leaves $(\mathbf{E}$ and $\mathbf{F})$ of the lettuce plants grown under several light treatments using monochromatic (M) light-emitting diodes (LEDs) (red, R; blue, B) at different stages. $\mathrm{R}$ and $\mathrm{B}$ indicate continuous irradiation of each of these lights for 4 weeks, and M1, $\mathrm{M} 2$, and M3 indicate changing from red to blue light at 1,2, or 3 weeks after the onset of the treatments, respectively. The data are means $\pm \mathrm{SE}(n=4)$. Different small caps indicate significant differences at $P=0.05(*), P=0.01(* *)$, and $P=0.001(* *)$.

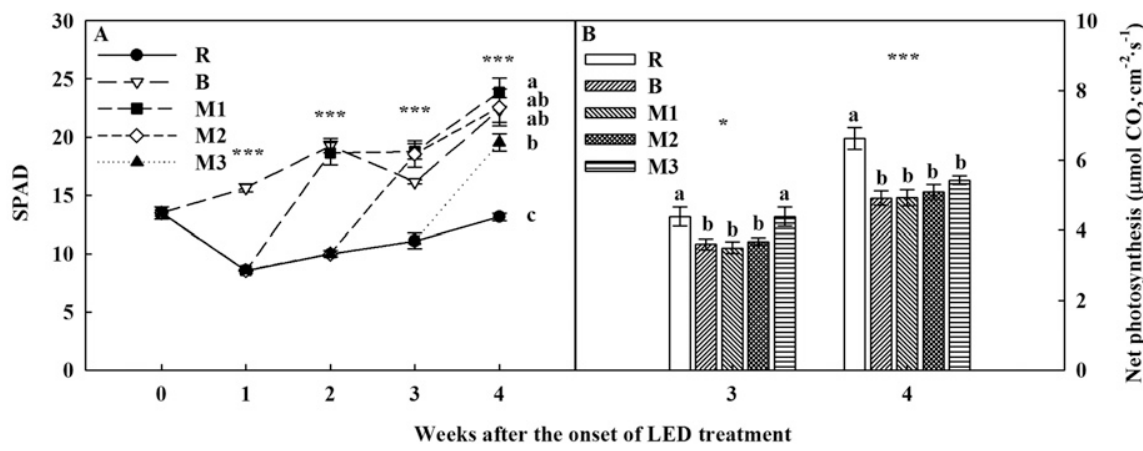

Fig. 2. SPAD (A) and net photosynthesis (B) of the lettuce plants grown under the monochromatic (M) light treatments (red, R; blue, B) at different stages. $\mathrm{R}$ and B indicate the continuous irradiation of each of these lights during 4 weeks of the treatment, and M1, M2, and M3 indicate changes from red to blue light at 1,2 , or 3 weeks after the onset of the treatments, respectively. The data are means $\pm \operatorname{SE}(n=4)$. Different small caps indicate significant differences at $P=0.05(*)$ and $P=0.001(* * *)$.

Japan) for 4 weeks after the onset of LED treatments. The PLAs obtained in each treatment were used to calculate the planting density and the total phenolic content per unit area (square meters).

\section{Secondary metabolites}

Lyophilized shoots were ground using a blender (MFM-002H; Hibell, Hwaseong, Korea) and stored at $4{ }^{\circ} \mathrm{C}$ until they were with minor modifications. A portion of about $40 \mathrm{mg}$ of each ground sample was extracted using $4 \mathrm{~mL}$ of $80 \%$ acetone for 15 min with ultrasonication (Sk5210HP; Hangzhou Nade Scientific Instrument, Zhejiang, China). Each sample extract was then centrifuged at 13,000 $g_{n}$ for $5 \mathrm{~min}$ at room temperature, and the supernatant was used in the subsequent analyses. Total phenolic concentrations and antioxidant capacity were determined as described in Son and Oh $(2013,2015)$ and expressed as the gallic acid-equivalent (GAE; mg) and the troloxequivalent antioxidant capacity (TEAC; mM) per shoot DW (g) (mg GAE. $\mathrm{g}^{-1}$ shoot DW and mM TEAC. $\mathrm{g}^{-1}$ shoot $\left.\mathrm{DW}\right)$, respectively.

Individual phenolic compounds. About $100 \mathrm{mg}$ of each ground sample was mixed with $1 \mathrm{~mL}$ of acidified acetonitrile $(0.5 \% \mathrm{v} / \mathrm{v}$ $\mathrm{HCl}$ ). This mixture was hydrolyzed in a water bath at $80{ }^{\circ} \mathrm{C}$ and sonicated for $30 \mathrm{~min}$ (Sk5210HP ultrasonicator; Hangzhou Nade Scientific Instrument). After centrifuging at $3000 g_{\mathrm{n}}$ for $20 \mathrm{~min}$ and filtering through a $0.22-\mu \mathrm{m}$ syringe filter (Noble Bio, Hwaseong, Korea), each sample's supernatant was used in the analysis of polyphenol compounds. These were characterized using a YL9100 high-performance liquid chromatography system (HPLC; Younglin, Anyang, Korea) and separated on an ACE AQ column $(4.6 \mathrm{~mm} \times$ $250 \mathrm{~mm}, 5 \mu \mathrm{m}$; Advanced Chromatography Technologies, Aberdeen, UK) equipped with a guard column. The column temperature and injection volume were set to $30{ }^{\circ} \mathrm{C}$ and $10 \mu \mathrm{L}$, respectively, and $100 \%$ acetonitrile and $0.5 \%$ acetic acid in water were used as solvents $\mathrm{A}$ and $\mathrm{B}$, respectively. The elution gradient was $0 \%$ to $10 \%$ A for $10 \mathrm{~min}, 10 \%$ to $20 \%$ A for $20 \mathrm{~min}, 20 \%$ to $30 \%$ A for 10 $\min , 30 \%$ to $40 \%$ A for $10 \mathrm{~min}, 40 \%$ to $80 \%$ A for $10 \mathrm{~min}, 80 \%$ to $0 \%$ A for $1 \mathrm{~min}$, and $0 \%$ to $0 \% \mathrm{~A}$ for $9 \mathrm{~min}$. The flow rate was $0.8 \mathrm{~mL} \cdot \mathrm{min}^{-1}$ and absorbance was recorded at $320 \mathrm{~nm}$. Calibration curves, expressed as milligrams per DW (g) $\left(\mathrm{mg} \cdot \mathrm{g}^{-1} \mathrm{DW}\right)$, were generated using standard chlorogenic, caffeic, chicoric, and ferulic acids, and kaempferol (all from Sigma-Aldrich, St. Louis, MO).

Gene expression. The youngest leaves of lettuces exposed to different treatments were collected every week and frozen with liquid nitrogen. Differences in the transcript levels of phenylalanine ammonialyase $(P A L)$ and chalcone synthase $(C H S)$, which are the key genes for the biosynthesis of phenolic compounds and flavonoids, respectively, were determined. Total RNA was isolated using the RNeasy ${ }^{\circledR}$ Plant Mini Kit (QIAGEN, Dusseldorf, Germany), and its concentration in each sample was determined using a DS-11 NanoDrop spectrophotometer (DeNovix, Wilmington, DE). Complementary DNA was synthesized from the RNA isolated from each sample using the QuantiTect Reverse Transcription Kit (QIAGEN). Quantitative real-time polymerase chain reaction (PCR) was performed in a Rotor-gene 6000 (Corbett Research, Mortlake, Australia) using 2× QuantiMix SYBR Kit (PhileKorea, 
Seoul, Korea) and a set of primers (Bioneer, Seoul, Korea) selected from the GenBank database (http://www.ncbi.nlm.nih.gov/ genbank/; Table 2). PCR conditions included an initial denaturation stage $\left(95^{\circ} \mathrm{C}\right.$, $10 \mathrm{~min}$ ), followed by 40 cycles of denaturation $\left(95{ }^{\circ} \mathrm{C}, 10 \mathrm{~s}\right)$, annealing $\left(52{ }^{\circ} \mathrm{C}\right.$ for $P A L$ and $55^{\circ} \mathrm{C}$ for $C H S, 15 \mathrm{~s}$ ), and extension $\left(72{ }^{\circ} \mathrm{C}, 20 \mathrm{~s}\right)$. The relative expression of RNA ( $\triangle \Delta C T$ value) was calculated using Rotor-gene 1.7 (Corbett Research, Sydney, Australia). Actin, a housekeeping gene, was used to normalize $P A L$ and $C H S$ transcript levels among treatments.

\section{Statistical analysis}

The experiment was conducted using a completely randomized design. All growth and secondary metabolite parameters were replicated four times, except for the measurements of PAL and CHS gene expression (three times). Reproducibility of the data presented here is supported by our previous studies (Son et al., 2012; Son and Oh, 2013). Analysis of variance and means comparison between treatments using Duncan's multiple range test were performed in SAS 9.2 (SAS Institute, Cary, $\mathrm{NC})$.

\section{Results}

\section{Monochromatic LEDs}

Growth characteristics. Lettuces irradiated with red light and then with blue light showed significant differences in growth characteristics (Fig. 1). All growth characteristics showed the highest values after irradiation with red light for 4 weeks and a pronounced growth inhibition after irradiation with blue light. Lettuce growth after 4 weeks of treatment decreased as the time of irradiation with blue light increased $(\mathrm{R}>$ M3 $>$ M2 $>$ M1 $>$ B), although M1 and M2 showed no significant difference irrespective of B. In all treatments, a rapid decline in lettuce growth was observed immediately after changing from red to blue light. During

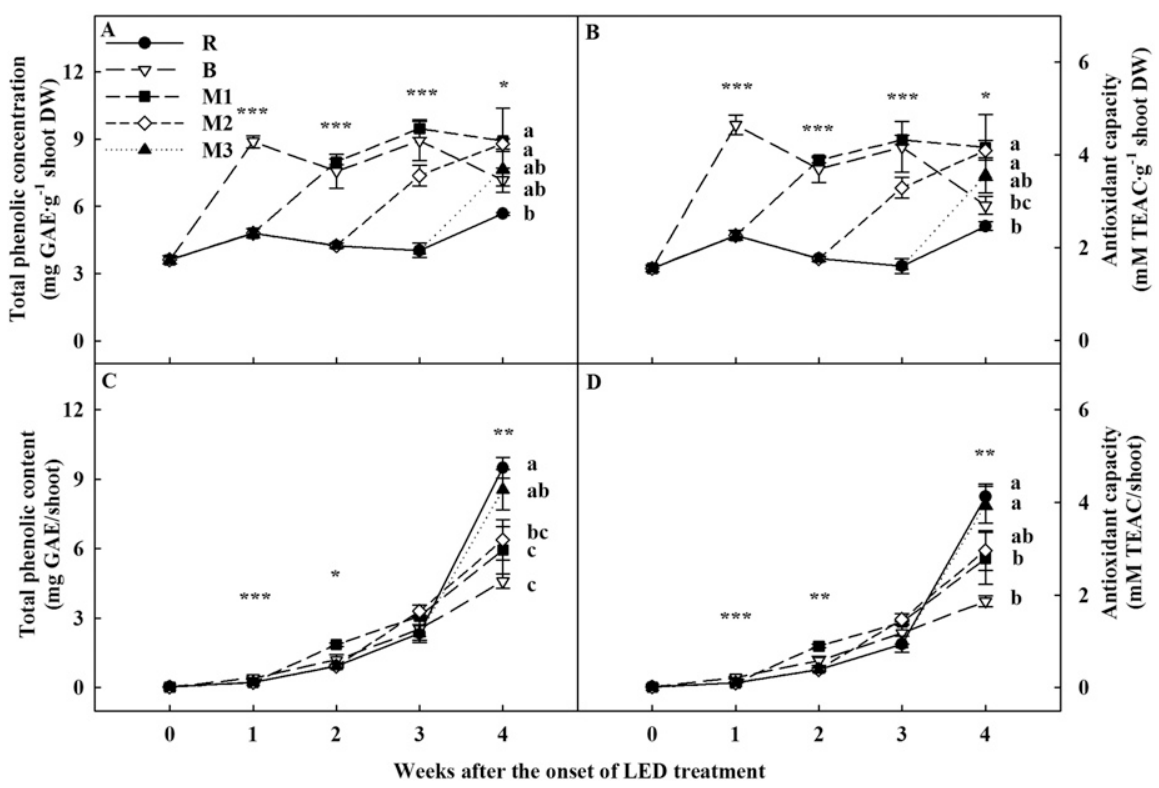

Fig. 3. Total phenolic concentrations and contents ( $\mathbf{A}$ and $\mathbf{C}$; left), and antioxidant capacity (B and $\mathbf{D}$; right) per unit of shoot dry weight (DW) (A and $\mathbf{B})$ and per plant $(\mathbf{C}$ and $\mathbf{D})$, obtained for the lettuce plants grown under several monochromatic (M) light treatments (red, R; blue, B) at different stages. B and R indicate the continuous irradiation of these lights for 4 weeks, and M1, M2, and M3 indicate changing from red to blue light at 1,2 , or 3 weeks after the onset of the treatments, respectively. The data are means $\pm \mathrm{SE}(n=4)$. Different small caps indicate significant differences at $P=0.05(*), P=0.01(* *)$, and $P=0.001(* * *)$. all growth stages, gradual significant differences were observed as blue irradiation increased.

Chlorophyll content and photosynthetic rate. Changing from red to blue light resulted in significant differences in lettuce's chlorophyll content and photosynthetic rate (Fig. 2). After 4 weeks of treatment, SPAD, an indirect index of chlorophyll content, was the lowest in the $\mathrm{R}$ treatment (Fig. 2A). Treatments using blue light (B, M1, M2, and M3) showed a significantly higher SPAD value compared with $\mathrm{R}$. A rapid increase in SPAD was observed in the week after the change from red to blue, at M1, M2, and M3. After 3 weeks of treatment, the photosynthetic rate was significantly higher (about 1.2 times) in plants exposed to red light ( $\mathrm{R}$ and $\mathrm{M} 3$ ) than it was in other treatments (Fig. 2B). At the fourth week, $R$ continued to show the highest photosynthetic rate among treatments, but the value in M3 decreased to a level similar to that of other treatments as it implied changing from red to blue light.

Total phenolic compounds and antioxidant capacity. Changing light quality from red to blue had a significant effect on total phenolic concentration and antioxidant capacity of lettuce leaves (Fig. 3). Total phenolic concentration showed a rapid increase after 1 week of irradiation in all treatments including blue light (B, M1, M2, and M3) and presented a significantly lower value in the $R$ treatment (Fig. 3A). Total phenolic contents showed the opposite trend as they were the highest in $\mathrm{R}$ followed by M3, M2, M1, and B (Fig. 3C). Antioxidant capacities per shoot DW and shoot (plant) showed a similar trend to that of total phenols (Fig. 3B and D). Blue irradiation affected the concentration of the phenolic and antioxidant compounds more sensitively in lettuce than that of contents.

Individual phenolic compounds. Changing light quality from red to blue affected both the concentration and the content of individual phenolic compounds such as chlorogenic, caffeic, chicoric, and ferulic acids, and kaempferol (Table 3). Considering phenolic concentration, chlorogenic acid concentration was significantly higher in M1, M2, and M3 (about 1.9 times) than in R. In addition, M3 induced the significantly highest concentration of chicoric acid among treatments. On the other hand, $\mathrm{R}$ induced the highest contents of caffeic and chicoric acids, whereas M3 induced the highest

Table 3. Concentrations and contents of individual phenolic compounds in the lettuce plants grown under five light treatments using monochromatic (M) LEDs (red, R; blue, B). R and B indicate the continuous irradiation of each of these lights for 4 weeks, and M1, M2, and M3 indicate changing from red to blue light at 1,2 , or 3 weeks after the onset of the treatments, respectively $(n=4)$.

$$
\text { Concn (mg g.shoot } \left.\mathrm{DW}^{-1}\right) \quad \text { Content (mg/shoot) }
$$

\begin{tabular}{|c|c|c|c|c|c|c|c|c|c|c|}
\hline Treatment & Chlorogenic acid & Caffeic acid & Chicoric acid & Ferulic acid & Kaempferol & Chlorogenic acid & Caffeic acid & Chicoric acid & Ferulic acid & Kaempferol \\
\hline $\mathrm{R}$ & $3.79 \mathrm{~b}^{\mathrm{z}}$ & 0.32 & $1.40 \mathrm{~b}$ & 0.03 & 0.03 & 6.38 & $0.53 \mathrm{a}$ & $2.36 \mathrm{a}$ & $0.05 \mathrm{~b}$ & 0.06 \\
\hline B & $6.65 \mathrm{ab}$ & 0.24 & $0.88 \mathrm{c}$ & 0.10 & 0.03 & 4.27 & $0.15 \mathrm{c}$ & $0.57 \mathrm{c}$ & $0.07 \mathrm{ab}$ & 0.02 \\
\hline M1 & $9.42 \mathrm{a}$ & 0.27 & $1.08 \mathrm{c}$ & 0.06 & 0.01 & 6.27 & $0.18 \mathrm{c}$ & $0.72 \mathrm{c}$ & $0.04 \mathrm{~b}$ & 0.01 \\
\hline M2 & $9.28 \mathrm{a}$ & 0.28 & $1.02 \mathrm{c}$ & 0.01 & 0.03 & 6.81 & $0.21 \mathrm{c}$ & $0.74 \mathrm{c}$ & $0.00 \mathrm{~b}$ & 0.02 \\
\hline M3 & $7.06 \mathrm{a}$ & 0.31 & $1.67 \mathrm{a}$ & 0.11 & 0.03 & 7.81 & $0.34 \mathrm{~b}$ & $1.84 \mathrm{~b}$ & $0.12 \mathrm{a}$ & 0.03 \\
\hline Significance ${ }^{y}$ & $* *$ & NS & $* * *$ & NS & NS & NS & $* * *$ & $* * *$ & $*$ & NS \\
\hline
\end{tabular}

LED = light-emitting diode.

${ }^{\mathrm{z}}$ Different lowercase letters within the columns indicate significant differences according to Duncan's multiple range test.

${ }^{\mathrm{y}} \mathrm{NS}=$ nonsignificant, $P=0.05(*), P=0.01(* *)$, and $P=0.001(* * *)$. 
contents of chlorogenic acid and the significantly highest ferulic acid values.

Gene expression. To assess the effect of changing light quality on the biosynthetic pathways for secondary metabolites, $P A L$ and $C H S$ gene expression was measured by the quantitative real-time PCR in lettuce leaves at each stage of light quality change from red to blue (Fig. 4). Changing from red to blue light increased the transcript levels of $P A L$ and $C H S$ at each stage, with the highest upregulations of these genes being observed at M1 (8.1 and 4.7 times, respectively).

\section{Combined LEDs}

Growth characteristics. Changing light quality in combined LEDs affected growth performance (Fig. 5). Among the control groups, i.e., plants continuously irradiated with R9B1, R8B2, and R6B4, the treatment comprising the highest percentage of blue light (R6B4) showed the lowest values in most growth characteristics, including shoot fresh weight, whereas R9B1 generally showed the highest values. Among the C treatments, C3 (from R9B1 to R6B4 after 2 weeks) showed the lowest values in all growth characteristics except for leaf number. Although growth characteristics under $\mathrm{C} 1, \mathrm{C} 2$, and $\mathrm{C} 4$ treatments were slightly distinct, a significant difference was not observed between these treatments. On the other hand, leaf number was similar to all treatments except R6B4.

Chlorophyll content and photosynthetic rate. Changing light quality in combined LEDs affected SPAD and photosynthetic rate during several growth stages of lettuces (Fig. 6). At 3 and 4 weeks of treatment, SPAD was not significantly different among treatments; however, the low SPAD value seen in R9B1 significantly increased after irradiation with R8B2 (C1) and R6B4 (C3) LEDs, which contained a larger fraction of blue (Fig. 6A). After 3 weeks, net photosynthesis was significantly higher under R9B1, $\mathrm{C} 2$, and $\mathrm{C} 4$ than it was in other treatments. All treatments showed lower net photosynthesis than R9B1 after 4 weeks of LED irradiation, with R6B4 presenting the lowest value (Fig. 6B).

Total phenolic compounds and antioxidant capacity. The effect of changing light quality in combined LED treatments on the total phenolic concentration and antioxidant capacity is shown in Fig. 7. Total phenolic concentration showed the lowest level in the R9B1 treatment, which contained the smallest fraction of blue light. On the other hand, changing light quality from R9B1 to R8B2 or R6B4 after 2 or 3 weeks of treatment resulted in the rapid increase of the total phenolic concentration, with the increasing rate being higher in R6B4 (41\%) than in R8B2 (24\%). However, when comparing the phenolic contents per shoot (plant), all treatments except R6B4 showed no significant difference after 4 weeks (Fig. 7C). Antioxidant capacity exhibited a similar trend (Fig. 7B and D).

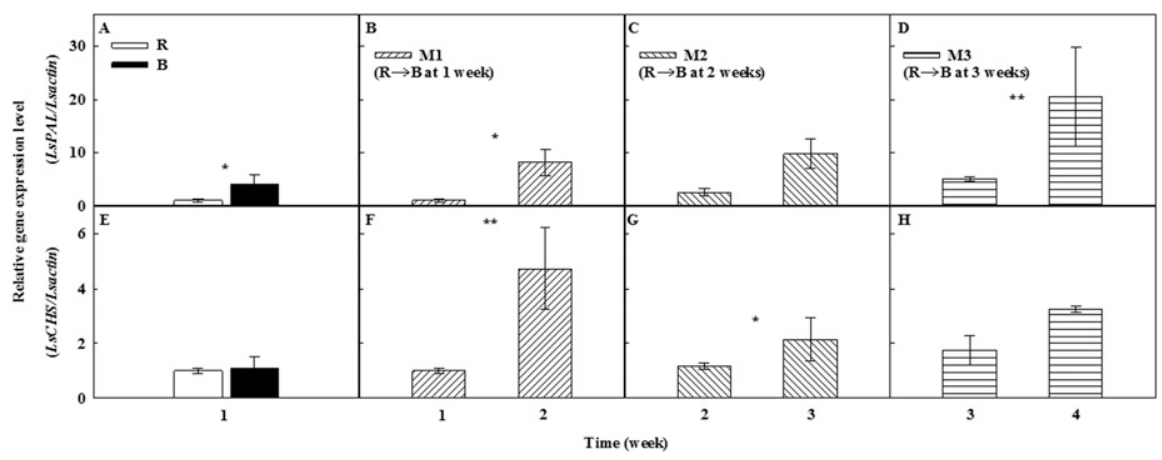

Fig. 4. Expression of $P A L$ (phenylalanine ammonia-lyase; $\mathbf{A}-\mathbf{D}$ ) and $C H S$ (chalcone synthase; $\mathbf{E}-\mathbf{H}$ ) genes in the lettuce plants grown under five monochromatic light treatments, determined 1 week after the onset of the experiments (A and $\mathbf{E}$ ) and 1 week after changing from red to blue light (B-D and $\mathbf{F}-\mathbf{H})$. Expression levels of $L s P A L$ and $L s C H S$ were normalized using actin (Lsactin), which is a housekeeping gene. Numbers below the columns indicate the week at which gene expression was determined under red light irradiation. The data are means $\pm \mathrm{SE}(n=3)$. Significance at $P=0.05(*)$ and significance at $P=0.01(* *)$.

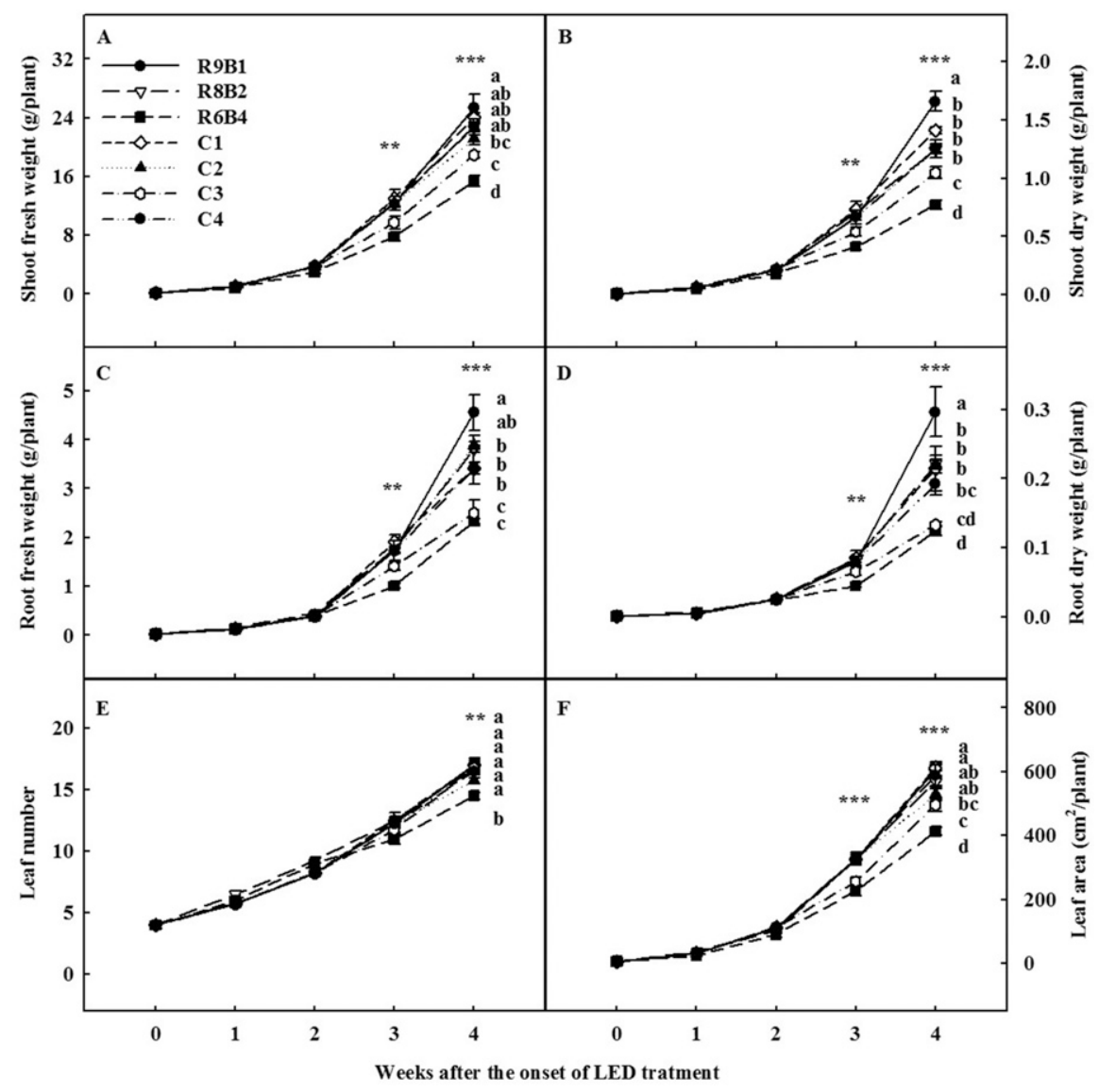

Fig. 5. Growth of shoots ( $\mathbf{A}$ and $\mathbf{B})$, roots $(\mathbf{C}$ and $\mathbf{D})$, and leaves $(\mathbf{E}$ and $\mathbf{F})$ of the lettuce plants grown under seven combined $(\mathbf{C})$ light treatments at different stages. R9B1, R8B2, and R6B4 indicate the continuous irradiation of each of these combined lights for 4 weeks. C1, C2, C3, and C4 indicate changing from R9B1 to R8B2 or R6B4 at 2 or 3 weeks after the onset of the treatments, respectively. The data are means \pm SE $(n=4)$. Different small caps above the bars indicate significant differences at $P=0.01(* *)$ and $P=0.001(* * *)$.

Individual phenolic compounds. Changing light quality from $\mathrm{R} 9 \mathrm{~B} 1$ to $\mathrm{R} 8 \mathrm{~B} 2$ or R6B4 affected lettuce's concentration of chlorogenic, caffeic, chicoric, and ferulic acids, and kaempferol (Table 4). Chlorogenic acid concentration was not significantly different among treatments, although higher values were obtained with larger proportions of blue-light irradiation (R6B4 $>$ R8B2 > $\mathrm{R} 9 \mathrm{~B} 1$ ). In addition, regardless of the time at which light-quality change was performed, increasing blue light irradiation (changing from R9B1 to R8B2 or R6B4; $\mathrm{C} 1-\mathrm{C} 4)$ led to an increase in individual 


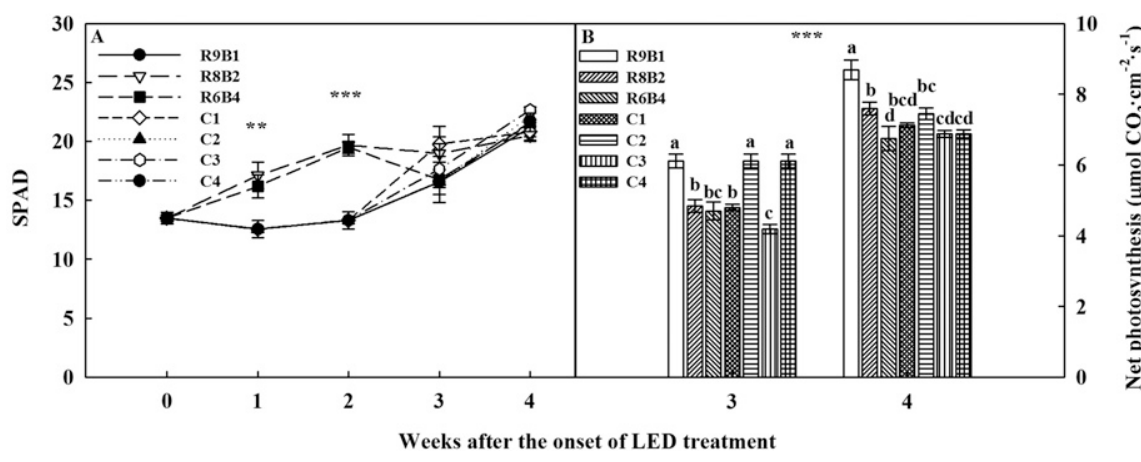

Fig. 6. SPAD (A) and net photosynthesis (B) obtained for the lettuce plants grown under several combined (C) light treatments at different stages. R9B1, R8B2, and R6B4 indicate the continuous irradiation of these combined lights for 4 weeks. C1, C2, C3, and C4 indicate changing from R9B1 to R8B2 or R6B4 at 2 or 3 weeks after the onset of the treatments, respectively. The data are means $\pm \operatorname{SE}(n=4)$. Different small caps indicate significant differences at $P=0.01(* *)$ and $P=0.001(* * *)$.

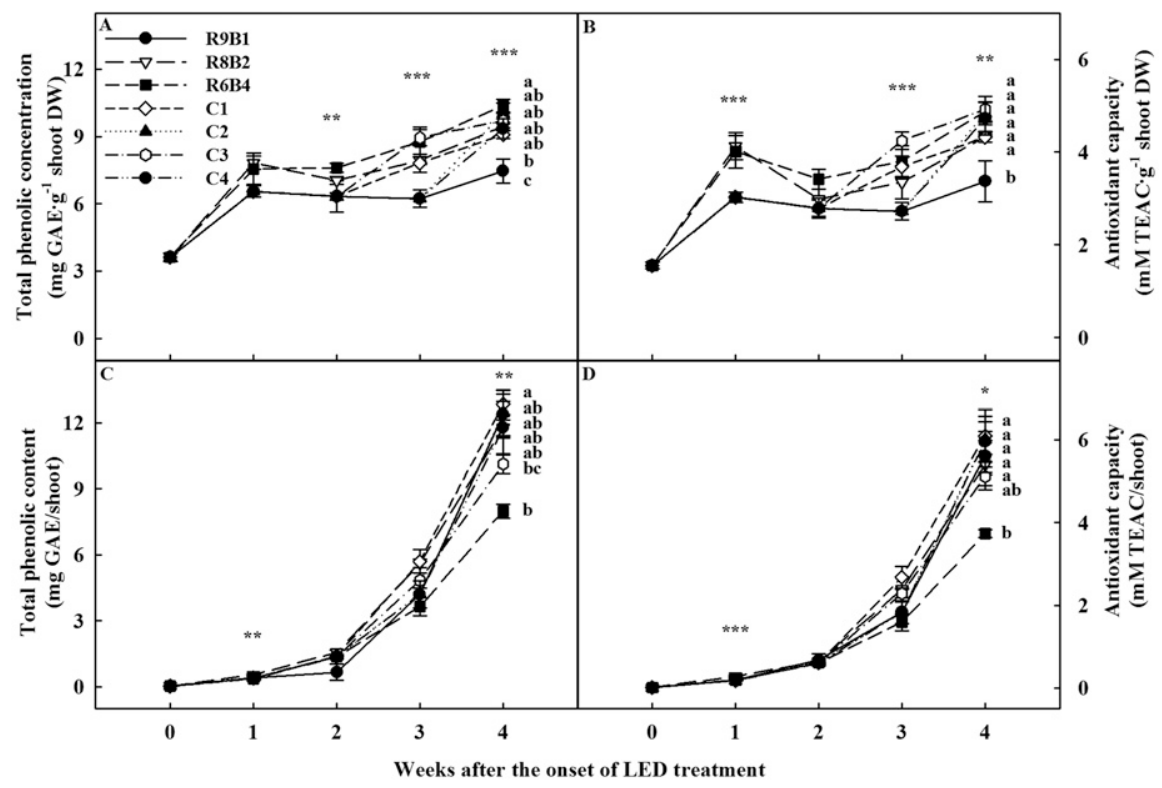

Fig. 7. Total phenolic concentrations and contents (A and $\mathbf{C}$; left), and antioxidant capacity (B and $\mathbf{D} ;$ right) per unit of shoot dry weight (DW) (A and $\mathbf{B}$ ) and per plant (C and $\mathbf{D})$ obtained for the lettuce plants grown under several combined (C) light treatments at different stages. R9B1, R8B2, and R6B4 indicate the continuous irradiation of these combined lights for 4 weeks. $\mathrm{C} 1, \mathrm{C} 2, \mathrm{C} 3$, and $\mathrm{C} 4$ indicate changing from R9B1 to R8B2 or R6B4 at 2 or 3 weeks after the onset of the treatments, respectively. The data are means \pm SE $(n=4)$. Different small caps indicate significant differences at $P=0.05(*), P=0.01(* *)$, and $P=0.001(* * *)$.

phenolic compounds. For chicoric acid, all C treatments showed a significantly higher value than R9B1 (>1.2 times). However, when considering the phenolic contents per shoot (plant), those subjected to the R9B1 treatment showed high values of all phenolic acids, and those subjected to $\mathrm{C} 1$ and $\mathrm{C} 4$ showed no significant differences when compared with R9B1, except for caffeic acid.

\section{Discussion}

Growth characteristics. Previous studies have shown that monochromatic red light was efficient for the growth of shoots and roots (Son et al., 2012) and that increased ratios of red in combined red and blue irradiation also enhanced lettuce growth (Son and Oh, 2013). The present results support these conclusions, although no growth, with differences in growth performances depending on the stage at which red light is irradiated (Table 1).

Chlorophyll content and photosynthetic rate. An increasing fraction of blue light effectively increased SPAD, the index of chlorophyll content. This is in agreement with the Banaś et al.'s (2012) report on blue light leading to the formation of chlorophyll, with the effective increase in chlorophyll formation resulting from monochromatic blue LED irradiation, and with the increase in SPAD resulting from increased ratios of blue light in combined LEDs (Son et al., 2012; Son and Oh, 2013). However, SPAD values after 4 weeks of irradiation were similar for all monochromatic treatments except R (Fig. 2A) and showed no significant differences among the combined treatments including blue light (Fig. 6A). These results imply that the qualitative effects of blue light are larger than its quantitative effects and that blue light is important for chlorophyll biosynthesis (Hogewoning et al., 2010).

Red light, on the other hand, had an effect on the photosynthetic rates similar to that registered for growth, suggesting a correlation between these two parameters (Kumagai et al., 2009). However, Kim et al. (2004) and Son and Oh (2015) found no correlation between the photosynthetic rate and growth values of lettuce leaves irradiated with an electrical lighting source installed in a leaf cuvette. This discrepancy might be explained by the setup used in the present study to measure photosynthesis: lettuce leaves were maintained under each lighting conditions for 4 weeks after transplanting, and photosynthesis was measured in a transparent leaf chamber (model 6400-08; LI-COR). Thus, studies evaluating light quality and photosynthesis under similar light conditions are necessary to obtain accurate data on photosynthetic rates in the plants' growth environment.

Secondary metabolites. In contrast to the growth results, the fraction of blue light irradiated was effective on the accumulation of secondary metabolites. Son et al. (2012) reported an enhancement in phenolic concentration and antioxidant capacity resulting from the PAL gateway enzyme activation in the biosynthesis of phenolics induced by monochromatic blue LEDs. In addition, the increase in phenolic concentration and antioxidant capacity accompanying the increase in the fraction of blue light in combined LEDs suggested that blue light within the visible wavelength is important for the production of secondary metabolites in lettuce (Son and Oh, 2013). The results obtained here for individual phenolic compounds were consistent with those of Taulavuori et al. (2016), who observed that enhancing blue light during light periods increased the concentrations of many bioactive compounds in lettuce, including chicoric acid. This effect has been reported in several lettuce cultivars (Li and Kubota, 2009; Ouzounis et al., 2015). Thus, blue light has been referred to 
Table 4. Concentrations and contents of individual phenolic compounds in the lettuce plants grown under seven light treatments using combined (C) LEDs. R9B1, R8B2, and R6B4 indicate the continuous irradiation of each of these lights for 4 weeks. C1, C2, C3, and C4 indicate changing from R9B1 to R8B2 or R6B4 at 2 or 3 weeks after the onset of treatments, respectively $(n=4)$.

\begin{tabular}{|c|c|c|c|c|c|c|c|c|c|c|}
\hline \multirow[b]{2}{*}{ Treatment } & \multicolumn{5}{|c|}{ Concentration (mg g.shoot $\mathrm{DW}^{-1}$ ) } & \multicolumn{5}{|c|}{ Content (mg/shoot) } \\
\hline & Chlorogenic acid & Caffeic acid & Chicoric acid & Ferulic acid & Kaempferol & Chlorogenic acid & Caffeic acid & Chicoric acid & Ferulic acid & $\overline{\text { Kaempferol }}$ \\
\hline$\overline{\mathrm{R} 9 \mathrm{~B} 1}$ & 7.59 & 0.58 & $1.74 \mathrm{c}^{\mathrm{z}}$ & 0.09 & 0.03 & $12.61 \mathrm{a}$ & $0.95 \mathrm{a}$ & $2.89 \mathrm{a}$ & $0.15 \mathrm{a}$ & $0.05 \mathrm{a}$ \\
\hline R8B2 & 8.71 & 0.47 & $1.94 \mathrm{bc}$ & 0.11 & 0.03 & $10.84 \mathrm{ab}$ & $0.58 \mathrm{bc}$ & $2.42 \mathrm{ab}$ & $0.14 \mathrm{a}$ & $0.04 \mathrm{abc}$ \\
\hline R6B4 & 9.35 & 0.45 & $2.00 \mathrm{abc}$ & 0.10 & 0.03 & $7.22 \mathrm{c}$ & $0.35 \mathrm{~d}$ & $1.54 \mathrm{c}$ & $0.08 \mathrm{~b}$ & $0.03 \mathrm{c}$ \\
\hline $\mathrm{C} 1$ & 9.24 & 0.49 & $1.97 \mathrm{bc}$ & 0.10 & 0.04 & $13.04 \mathrm{a}$ & $0.70 \mathrm{~b}$ & $2.77 \mathrm{a}$ & $0.14 \mathrm{a}$ & $0.05 \mathrm{ab}$ \\
\hline $\mathrm{C} 2$ & 9.35 & 0.47 & $2.25 \mathrm{a}$ & 0.11 & 0.03 & $11.59 \mathrm{ab}$ & $0.58 \mathrm{bc}$ & $2.79 \mathrm{a}$ & $0.14 \mathrm{a}$ & $0.03 \mathrm{bc}$ \\
\hline $\mathrm{C} 3$ & 8.52 & 0.42 & $2.03 \mathrm{ab}$ & 0.07 & 0.03 & $8.90 \mathrm{bc}$ & $0.44 \mathrm{~cd}$ & $2.13 \mathrm{~b}$ & $0.07 \mathrm{~b}$ & $0.04 \mathrm{abc}$ \\
\hline $\mathrm{C} 4$ & 8.44 & 0.44 & $2.11 \mathrm{ab}$ & 0.08 & 0.03 & $10.66 \mathrm{ab}$ & $0.55 \mathrm{bc}$ & $2.66 \mathrm{ab}$ & $0.10 \mathrm{ab}$ & $0.04 \mathrm{ab}$ \\
\hline Significance ${ }^{\mathrm{y}}$ & NS & NS & $*$ & NS & NS & $* *$ & $* * *$ & $* * *$ & $* *$ & $*$ \\
\hline
\end{tabular}

LED = light-emitting diode.

${ }^{\mathrm{z}}$ Different lowercase letters within the columns indicate significant differences according to Duncan's multiple range test.

${ }^{\mathrm{y}}$ Nonsignificant (NS), $P=0.05(*), P=0.01(* *)$, and $P=0.001(* * *)$.
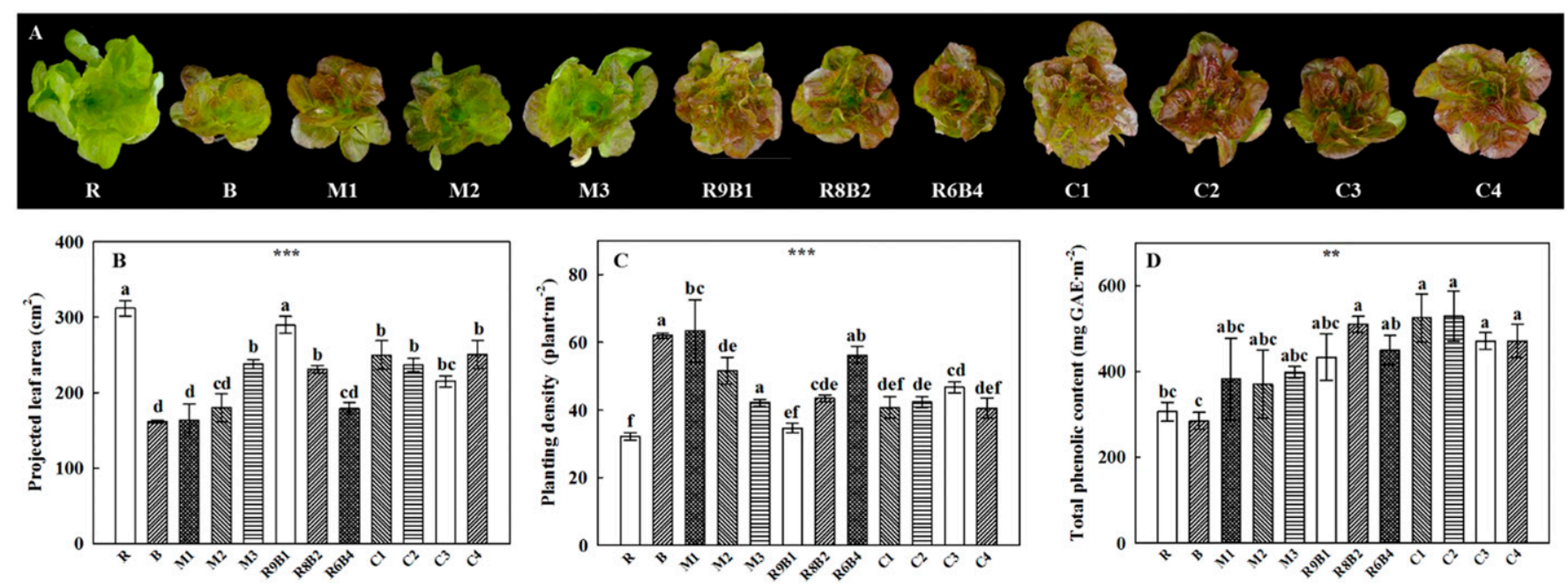

Fig. 8. Lettuce plants (A), projected leaf area (B), planting density (C), and total phenolic content (D) of the lettuce plants grown under several treatments using monochromatic and combined lights. R and B indicate the continuous irradiation of red and blue light, respectively, for 4 weeks. M1, M2, and M3 indicate changes from red to blue light at 1,2, or 3 weeks after the onset of treatments, respectively. R9B1, R8B2, and R6B4 indicate the continuous irradiation of these combined lights for 4 weeks. C1, C2, C3, and C4 indicate changing from R9B1 to R8B2 or R6B4 at 2 or 3 weeks after the onset of treatments, respectively. The data are means \pm SE $(n=4)$. Different small caps indicate significant differences at $P=0.01(* *)$ and $P=0.001(* * *)$.

generally induce the biosynthesis of secondary metabolites in crops. This phenomenon might be explained by the induction of reactive oxygen species in plants exposed to blue light, which has a relatively shorter wavelength than red light, and is considered a metabolism similar to the accumulation of secondary metabolites by ultraviolet light (Kuse et al., 2014; Lee et al., 2013). However, changing light quality in monochromatic treatments led to more rapid changes in growth and secondary metabolites than in treatments using combined red and blue light, suggesting that metabolic processes are more sensitive to changes in a particular light (red $\rightarrow$ blue; M1, M2, and M3) than to changes in the ratios of red/blue light $(\mathrm{C} 1$, $\mathrm{C} 2, \mathrm{C} 3$, and $\mathrm{C} 4)$. This response between monochromatic lights and combined lights may be associated with metabolic changes induced by the distinct activation of photoreceptors such as phytochromes and cryptochromes effectively absorbing red and blue lights, respectively (Jiao et al., 2007). However, further studies would be required for in-depth understanding.

PAL and CHS, which are synthesized via the phenylpropanoid and flavonoid pathway, are the important enzymes for the biosynthesis of phenols and flavonoids (Koukol and Conn, 1961; Park et al., 2007). Activation of $P A L$ and $C H S$ gene expressions supported a positive effect of blue light on secondary metabolites (Fig. 7), with the accumulation of flavonoids showing a similar trend to that found for total phenolic concentration and antioxidant capacity (data not shown). Previous studies also found that the expressions of PAL gene and PAL enzyme in lettuce were stimulated by blue light (Heo et al., 2012; Son et al., 2012). These results suggest that the biosynthetic pathways of secondary metabolites, such as phenols and flavonoids, might be simultaneously activated in lettuces irradiated with different light qualities.

On the other hand, the productivity (biomass) enhanced by red light largely influenced the content, but not the concentration, of the secondary metabolites produced by plants. As such, changing light quality (M1-M3 and C1-C4) was not effective for enhancing the content of secondary metabolites. However, plant density is also important when considering plants' production in a limited area, as suggested by the PLA, plant density, and total phenolic content obtained here (Fig. 8). Total phenolic content per unit area was higher in the treatments changing light quality (M1-M3 and $\mathrm{C} 1-\mathrm{C} 4)$ than it was in the continuously irradiated controls (R, B, R9B1, R8B2, and R6B4) (Fig. 8D). In fact, this effect was more sensitive to monochromatic than it was to combined light. M1, M2, and M3 showed a similar level of total phenolic content to that of combined LEDs (R9B1, R8B2, and R6B4), suggesting that monochromatic and combined light might have a similar effect on secondary metabolites production. Moreover, irradiation with combined LEDs induced an increase in growth and secondary metabolite accumulation and total phenolic content per unit area. Hence, the light-quality changes performed in this study were effective to maximize the production of secondary metabolites in optimum plant densities within a limited area.

Conclusion. The growth rate and biosynthesis of secondary metabolites observed in lettuce at different stages clearly responded to changes in light quality, with red light being the most effective on growth and blue light being the most effective on the 
biosynthesis of secondary metabolites. As this study was conducted using monochromatic and combined LEDs under the same environmental conditions and during the same period, it enabled comparisons between these two light sources, which were not possible in our previous studies (Son et al., 2012; Son and Oh, 2013). Thus, it was possible to verify that combined light induced larger rates of growth and accumulation of secondary metabolites than monochromatic light. This clear difference was also corroborated by physiological (plant size), morphological (leaf shape), and accumulation of secondary metabolites (leaf pigment) data. These results suggest that light changes can be used in commercial plant cultivation areas that target bioactive compounds. This study also suggested that changing light quality using different LEDs or a combination of LEDs to enhance the productivity (growth) and quality (secondary metabolites) of crops might be a meaningful technique to produce higher quality and lessexpensive crops.

\section{Literature Cited}

Ainsworth, E.A. and K.M. Gillespie. 2007. Estimation of total phenolic content and other oxidation substrates in plant tissues using Folin-Ciocalteu reagent. Nat. Protoc. 2:875-877.

Banaś, A.K., C. Aggarwal, J. Labuz, O. Sztatelman, and H. Gabryś. 2012. Blue light signaling in chloroplast movements. J. Expt. Bot. 63:1559-1574.

Bian, Z.H., Q.C. Yang, and W.K. Liu. 2014. Effects of light quality on the accumulation of phytochemicals in vegetables produced in controlled environments: A review. J. Sci. Food Agr. 95:869-877.

Bryant, J.P., F.S. Chapin, and D.R. Klein. 1983. Carbon/nutrient balance of boreal plants in relation to vertebrate herbivory. Oikos 40:357-368.

Carvalho, S.D. and K.M. Folta. 2014. Sequential light programs shape kale (Brassica napus) sprout appearance and alter metabolic and nutrient content. Hort. Res. 1:8, doi: 10.1038/ hortres.2014.8.

Gartia, S., M.K. Pradhan, P.N. Joshi, U.C. Biswal, and B. Biswal. 2003. UV-A irradiation guards the photosynthetic apparatus against UV-Binduced damage. Photosynthetica 41:545-549. Heo, J.W., D.H. Kang, H.S. Bang, S.G. Hong, C. Chun, and K.K. Kang. 2012. Early growth, pigmentation, protein content, and phenylalanine ammonia-lyase activity of red curled lettuces grown under different lighting conditions. Kor. J. Hort. Sci. Technol. 30:6-12.

Herms, D.A. and W.J. Mattson. 1992. The dilemma of plants: To grow or defend. Qrtly. Rev. Biol. 67:283-335.

Hogewoning, S.W., G.H. Maljaars, H. Poorter, W. van Ieperen, and J. Harbinson. 2010. Blue light dose-responses of leaf photosynthesis, morphology, and chemical composition of $\mathrm{Cucu}$ mis sativus grown under different combinations of red and blue light. J. Expt. Bot. 61:31073117.

Jiao, Y., O.S. Lau, and X.W. Deng. 2007. Lightregulated transcriptional networks in higher plants. Nat. Rev. Genet. 8:217-230.

Jishi, T., K. Kimura, R. Matsuda, and K. Fujiwara. 2016. Effects of temporally shifted irradiation of blue and red LED light on cos lettuce growth and morphology. Sci. Hort. 198:227-232.

Kim, H.H., G.D. Goins, R.M. Wheeler, and J.C. Sager. 2004. Green-light supplementation for enhanced lettuce growth under red- and blue-light-emitting diodes. HortScience 39:1617-1622.

Koukol, J. and E.E. Conn. 1961. The metabolism of aromatic compounds in higher plants. IV. Purification and properties of the phenylalanine deaminase of Hordeum vulgare. J. Biol. Chem. 236:2692-2698.

Kumagai, E., T. Araki, and F. Kubota. 2009. Correlation of chlorophyll meter readings with gas exchange and chlorophyll fluorescence in flag leaves of rice (Oryza sativa L.) plants. Plant Prod. Sci. 12:50-53.

Kuse, Y., K. Ogawa, K. Tsuruma, M. Shimazawa, and H. Hara. 2014. Damage of photoreceptorderived cells in culture induced by light emitting diode-derived blue light. Sci. Rpt. 4:5223, doi: $10.1038 /$ srp05223.

Lee, M.-J., J.E. Son, and M.-M. Oh. 2013. Growth and phenolic compounds of Lactuca sativa $\mathrm{L}$. grown in a closed-type plant production system with UV-A, -B, or -C lamp. J. Sci. Food Agr. 94:197-204

Lefsrud, M., D. Kopsell, and C. Sams. 2008. Irradiance from distinct wavelength light-emitting diodes affect secondary metabolites in kale. HortScience 43:2243-2244.
Li, Q. and C. Kubota. 2009. Effects of supplemental light quality on growth and phytochemicals of baby leaf lettuce. Environ. Expt. Bot. 67:5964.

Massa, G., H. Kim, R. Wheeler, and C. Mitchell. 2008. Plant productivity in response to LED lighting. HortScience 43:1951-1956.

Miller, N.J. and C.A. Rice-Evans. 1996. Spectrophotometric determination of antioxidant activity. Redox Rep. 2:161-171.

Olle, M. and A. Viršile. 2013. The effects of lightemitting diode lighting on greenhouse plant growth and quality. Agr. Food Sci. 22:223234

Ouzounis, T., B.R. Parjikolaei, X. Fretté, E. Rosenqvist, and C.-O. Ottosen. 2015. Predawn and high intensity application of supplemental blue light decreases the quantum yield of PSII and enhances the amount of phenolic acids, flavonoids, and pigments in Lactuca sativa. Front. Plant Sci. 6:19, doi: 0.3389/fpls.2015.00019.

Park, J.-S., M.-G. Choung, J.-B. Kim, B.-S. Hahn, J.-B. Kim, S.-C. Bae, K.-H. Roh, Y.-H. Kim, C.-I. Cheon, M.-K. Sung, and K.-J. Cho. 2007. Genes up-regulated during red coloration in UV-B irradiated lettuce leaves. Plant Cell Rpt. 26:507-516.

Piovene, C., F. Orsini, S. Bosi, R. Sanoubar, V. Bregola, G. Dinelli, and G. Gianquinto. 2015. Optimal red:blue ratio in led lighting for nutraceutical indoor horticulture. Sci. Hort. 193:202-208.

Son, K.-H. and M.-M. Oh. 2013. Leaf shape, growth, and antioxidant phenolic compounds of two lettuce cultivars grown under various combinations of blue and red light-emitting diodes. HortScience 48:988-995.

Son, K.-H. and M.-M. Oh. 2015. Growth, photosynthetic and antioxidant parameters of two lettuce cultivars as affected by red, green, and blue light-emitting diodes. Hort. Environ. Biotechnol. 56:639-653.

Son, K.-H., J.-H. Park, D. Kim, and M.-M. Oh. 2012. Leaf shape, growth, and phytochemicals in two leaf lettuce cultivars grown under monochromatic light-emitting diodes. Kor. J. Hort Sci. Technol. 30:664-672.

Taulavuori, K., V. Hyöky, J. Oksanen, E. Taulavuori, and R. Julkunen-Tiitto. 2016. Species-specific differences in synthesis of flavonoids and phenolic acids under increasing periods of enhanced blue light. Environ. Expt. Bot. 121:145-150. 